\title{
Beneficial Regulation of Matrix Metalloproteinases for Skin Health
}

\author{
Neena Philips, ${ }^{1}$ Susan Auler, ${ }^{1}$ Raul Hugo, ${ }^{1}$ and Salvador Gonzalez ${ }^{2,3}$ \\ ${ }^{1}$ School of Natural Sciences, Fairleigh Dickinson University, H-DH4-03, 1000 River Road, Teaneck, NJ 07666, USA \\ ${ }^{2}$ Industrial Cantabria Farmaceutica, S.A, Madrid, Spain \\ ${ }^{3}$ Dermatology Service, Memorial Sloan-Kettering Cancer Center, NY 10065, USA \\ Correspondence should be addressed to Neena Philips, neenaphilips@optonline.net
}

Received 21 September 2010; Accepted 21 December 2010

Academic Editor: Yong-Doo Park

Copyright (c) 2011 Neena Philips et al. This is an open access article distributed under the Creative Commons Attribution License, which permits unrestricted use, distribution, and reproduction in any medium, provided the original work is properly cited.

Matrix metalloproteinases (MMPs) are essential to the remodeling of the extracellular matrix. While their upregulation facilitates aging and cancer, they are essential to epidermal differentiation and the prevention of wound scars. The pharmaceutical industry is active in identifying products that inhibit MMPs to prevent or treat aging and cancer and products that stimulate MMPs to prevent epidermal hyperproliferative diseases and wound scars.

\section{Introduction}

Matrix metalloproteinases (MMPs) are essential to the remodeling of the extracellular matrix. While their upregulation facilitates aging and cancer, they are essential to epidermal differentiation and the prevention of wound scars. The pharmaceutical industry is active in identifying products that inhibit MMPs to prevent or treat aging and cancer and products that stimulate MMPs to prevent epidermal hyperproliferative diseases and wound scars.

\section{Matrix Metalloproteinases}

Matrix metalloproteinases (MMPs) are a group of zincdependent extracellular proteinases, also called matrixins or collagenases, which remodel the extracellular matrix (ECM) [1-10]. The ECM gives tissue its structural integrity and predominantly comprises of the fibrillar collagens, basement membrane, and elastin fibers composed of elastin and fibrillin [11-13]. There are three predominant groups of MMPs: collagenases, gelatinases, and stromelysins [113]. The collagenases (MMP-1, -8, -13, and -18) cleave interstitial (structural) collagens, with MMP-1 as the predominant one. Gelatinases, primarily MMP-2 and MMP-9, degrade basement membrane collagens and degrade denatured structural collagens. The stromelysins (MMP-3, -10, -11, and -19) degrade basement membrane collagens as well as proteoglycans and matrix glycoproteins. The other MMP classes include membrane-type MMPs (MT-MMP: MMP-14, -15, -16, -17, -24, and -25) that activate MMPs, matrilysins that degrade the basement membrane (MMP-7 and -26), elastase that degrades primarily elastin (MMP-12), and others (MMP-20,-21, -22,-23,-27, and -28). MMPs are regulated in expression or activity at several different levels: gene expression, activation, and cellular inhibition of activity by tissue inhibitors of matrix metalloproteinases (TIMPs), especially TIMP-1 and TIMP-2 [12]. Epithelial cells, fibroblasts, neutrophils, and mast cells are some of the cell types that produce MMPs [1-13].

Transforming growth factor- $\beta$ (TGF- $\beta$ ) is a predominant regulator of the expression of MMPs and the ECM $[1,2,12]$. It is secreted extracellularly in a latent form and activated by proteases to form the mature TGF- $\beta$ that binds to its receptor complexes to activate Smads and thereby the regulation of various genes, including MMPs, collagen, and elastin $[1,2$, 12]. TGF- $\beta$ has differential effects in different cell types [ 1,2 , 12]. It inhibits MMP- 1 and stimulates collagen, MMP-2, and TIMPs in fibroblasts, whereas in keratinocytes it stimulates the expression of MMPs and inhibits cell growth $[1,2,12]$. 


\section{Skin Aging and Carcinogenic Effects of Matrix Metalloproteinases}

3.1. Skin Aging and Its Prevention. Skin aging is the result the intrinsic chronological aging process superimposed by environmental factors, predominantly exposure to ultraviolet (UV) radiation (photoaging) [1, 2, 11-17]. UV radiation shifts the cellular balance to oxidative stress, inflammation, immunosuppression, and inhibition of TGF- $\beta[1,2$, 12]. In addition, UV radiation stimulates proinflammatory and proangiogenic cytokines such as interleukin-1 (IL-1), interleukin-6 (IL-6), tumor necrosis factor- $\alpha$ (TNF- $\alpha$ ), and vascular endothelial growth factor (VEGF) [1, 2]. Cellular damage in photoaged skin includes cell loss, DNA damage, lipid peroxidation, and compromise of skin barrier function [13].

Alterations in collagen and elastin of the ECM are primarily responsible for the clinical manifestations of skin aging such as wrinkles, sagging, and laxity $[1,2,11-13$, $17,18]$. The atrophy of collagen and elastin fibers in skin aging is predominantly from the increased expression of their degradative enzymes, collagenases (MMP-1), gelatinases (MMP-2 and -9), and elastases [1-13]. Collagen fibers are degraded by MMP-1 and MMP-2 and the elastin fibers by elastases, MMP-2, and MMP-9 [12].

Natural products that have been identified in our laboratory to inhibit MMPs and elastase and simultaneously stimulate collagen and elastin are Polypodium leucotomos extract, lutein, and xanthohumol [12-18]. These products consist of polyphenols, carotenoids, or flavonoids with antioxidant, anti-inflammatory, photoprotective, or anticarcinogenic properties [12-18].

$P$. leucotomos is rich in polyphenols [1]. It directly inhibits activities of MMPs [12]. P. leucotomos inhibits expression of MMPs in epidermal keratinocytes and fibroblasts and stimulates fibrillar collagens, elastin, and TGF- $\beta$ in dermal fibroblasts $[12,13]$. In addition, it inhibits cellular oxidative stress and thereby membrane damage and lipid peroxidation [13]. Furthermore, it functions as a sunscreen [14, 15].

Lutein is a non-provitamin A carotenoid that inhibits epidermal hyperproliferation, expansion of mutated keratinocytes, and the infiltration of mast cells in response to solar radiation, and thereby photoaging [16]. The mechanism to lutein's antiaging and antiphotoaging effects includes the inhibition of MMP-to-TIMP ratio in dermal fibroblasts and the inhibition of cell loss and membrane damage in ultraviolet radiation-exposed fibroblasts [17].

Xanthohumol, a flavonoid, directly inhibits MMPs (-1, -3 , and -9) and elastase activities while dramatically increasing the expression of types I, III, and V collagens, elastin, fibrillin-1, and fibrillin-2 in dermal fibroblasts [18].

3.2. Skin Cancer and Its Prevention. Carcinogenesis is characterized by development of tumors from genetic alterations and immunosuppression. The mechanisms also include oxidative stress from reactive oxygen species, inflammation and DNA damage [19]. Malignant tumors metastasize, with MMPs playing a central role [19]. Cancer invasion and metastasis of various cancer types parallelly increased expression of MMPs [19]. MMPs activate growth factors such as TGF- $\beta$ and VEGF to induce angiogenesis [19]. Furthermore, MMPs contribute to cancer progression by degrading the ECM, basement membrane, and E-cadherin molecules that hold cells together [19-22]. In our laboratory we have investigated two categories of agents that may prevent or facilitate carcinogenesis through the regulation of expression of MMPs: (a) plant extracts or vitamins and (b) hormones.

The plant extracts or vitamins examined in our laboratory for MMP regulation in cancer cells are P. leucotomos, lutein and ascorbic acid $[12,17,23,24]$. P. leucotomos inhibits MMP-1 expression transcriptionally, through AP1 promoter sequence, and stimulates the expression of TIMP-2 in melanoma cells [4]. P. leucotomos in addition inhibits TGF- $\beta$, known to stimulate MMPs in cancer cells [12]. In photo-carcinogenesis experiments with rats, lutein inhibits tumor multiplicity, volume, and tumor-free survival time [16]. Furthermore, lutein inhibits MMP-1 and stimulates TIMPs $(-1$ and -2$)$, to reduce MMP/TIMP ratio and thereby carcinogenesis [17]. Ascorbate has dose dependent differential effects on cancer cell growth versus expression of MMPs/metastasis potential [23, 24]. At lower concentrations, ascorbate inhibits cell growth with dramatic increases in the expression of MMPs, which are inhibited by cotreatment with $P$. leucotomos or gene silencing with MMP siRNA [23, 24].

\section{Beneficial Effects of Matrix Metalloproteinases}

4.1. Epidermal Differentiation and Wound Repair. Hyperproliferative diseases of the skin are associated with reduced MMPs [25]. The expression of MMP-9 is reduced in psoriatic keratinocytes, with hyperproliferative keratinocytes [26]. Hyperproliferative skin diseases are also associated with reduced generation of ceramides (the major lipids of the stratum corneum) or increased ceramidase activity [27-29]. Ceramides are intracellular messengers of the sphingomyelin cycle that activate protein kinase C-alpha and stress-activated protein kinases to induce apoptosis and epidermal differentiation [30]. The experiments in our laboratory indicate that ceramide induces MMP-1 expression in keratinocytes through the activator protein-1 (AP-1) sequence and simultaneously inhibits keratinocyte cell viability by apoptosis [25]. The mechanism of MMP-1 gene regulation by ceramide may be through the stimulation of TGF- $\beta$, known to inhibit epidermal cell viability [25].

MMPs are essential to wound healing. They remove wound debris, facilitate epithelization, and prevent wound scars from excess collagen [31-34]. In our laboratory, copper is effective in inducing wound healing whereas the antibodies to TGF- $\beta$ associated with wound scars are ineffective [3134].

The biologically active concentrations of copper range from 1 to $200 \mu \mathrm{M}$ in tissue engineering for wound care, without toxicity [35]. The lower copper concentrations $(0.3-3 \mu \mathrm{M})$ stimulate activity of MMPs whereas the higher 
concentrations $(1-100 \mu \mathrm{M})$ stimulate the expression of MMPs in fibroblasts [31]. Adult wound scars are attributed to increased TGF- $\beta$ expression and subsequent collagen deposition [33]. However, TGF- $\beta$ antibodies cause feedback stimulation of TGF- $\beta[32,33]$. The feedback stimulation of TGF- $\beta$ in turn inhibits MMPs and stimulates TIMPs in fibroblasts, with further scarring potential $[32,33]$.

\section{Summary}

MMPs are potent proteases that remodel the ECM. It is central to the aging and cancer process. Polypodium leucotomos extract, lutein, and xanthohumol are effective in inhibiting expression and/or activities of MMPs, and thereby aging and cancer. Conversely, MMPs can prevent psoriasis and wound scars. Ceramide stimulates expression of MMP-1 and simultaneously inhibits cell viability. Copper stimulates MMPs, which may be its mechanism to improve wound healing. However, the antibodies to TGF- $\beta$ cause feedback stimulation of TGF- $\beta$, and further scarring potential.

\section{References}

[1] N. Philips, "Counteraction of skin inflammation and aging or cancer by polyphenols," in Anti-Inflammatory and Anti-Allergy Agents in Medicinal Chemistry, F. Epifano, Ed., Bentham Science, 2010.

[2] N. Philips, "Experimental physiology in anti-skin aging," in Skin Anatomy and Physiology Research Development, F. Columbus, Ed., Nova Science, 2009.

[3] C. W. Lee, H. J. Choi, H. S. Kim et al., "Biflavonoids isolated from Selaginella tamariscina regulate the expression of matrix metalloproteinase in human skin fibroblasts," Bioorganic and Medicinal Chemistry, vol. 16, no. 2, pp. 732-738, 2008.

[4] M. R. Khorramizadeh, E. E. Tredget, C. Telasky, Q. Shen, and A. Ghahary, "Aging differentially modulates the expression of collagen and collagenase in dermal fibroblasts," Molecular and Cellular Biochemistry, vol. 194, no. 1-2, pp. 99-108, 1999.

[5] A. J. T. Millis, M. Hoyle, H. M. McCue, and H. Martini, "Differential expression of metalloproteinase and tissue inhibitor of metalloproteinase genes in aged human fibroblasts," Experimental Cell Research, vol. 201, no. 2, pp. 373-379, 1992.

[6] A. Berton, G. Godeau, H. Emonard et al., "Analysis of the ex vivo specificity of human gelatinases $A$ and $B$ towards skin collagen and elastic fibers by computerized morphometry," Matrix Biology, vol. 19, no. 2, pp. 139-148, 2000.

[7] M. Brennan, H. Bhatti, K. C. Nerusu et al., "Metalloproteinase-1 is the major collagenolytic enzyme responsible for collagen damage in UV-irradiated human skin," Photochemistry and Photobiology, vol. 78, no. 1, pp. 43-48, 2003.

[8] N. Tsuji, S. Moriwaki, Y. Suzuki, Y. Takema, and G. Imokawa, "The role of elastases secreted by fibroblasts in wrinkle formation: implication through selective inhibition of elastase activity," Photochemistry and Photobiology, vol. 74, no. 2, pp. 283-290, 2001.

[9] F. Rijken, R. C. M. Kiekens, E. Van Den Worm, P. L. Lee, H. Van Weelden, and P. L. B. Bruijnzeel, "Pathophysiology of photoaging of human skin: focus on neutrophils," Photochemical and Photobiological Sciences, vol. 5, no. 2, pp. 184-189, 2006.
[10] F. Rijken, R. C. M. Kiekens, and P. L. B. Bruijnzeel, "Skininfiltrating neutrophils following exposure to solar-simulated radiation could play an important role in photoageing of human skin," British Journal of Dermatology, vol. 152, no. 2, pp. 321-328, 2005.

[11] N. Philips, D. Burchill, D. O’Donoghue, T. Keller, and S. Gonzalez, "Identification of benzene metabolites in dermal fibroblasts as nonphenolic: regulation of cell viability, apoptosis, lipid peroxidation and expression of matrix metalloproteinase 1 and elastin by benzene metabolites," Skin Pharmacology and Physiology, vol. 17, no. 3, pp. 147-152, 2004.

[12] N. Philips, J. Conte, Y. J. Chen et al., "Beneficial regulation of matrixmetalloproteinases and their inhibitors, fibrillar collagens and transforming growth factor- $\beta$ by Polypodium leucotomos, directly or in dermal fibroblasts, ultraviolet radiated fibroblasts, and melanoma cells," Archives of Dermatological Research, vol. 301, no. 7, pp. 487-495, 2009.

[13] N. Philips, J. Smith, T. Keller, and S. Gonzalez, "Predominant effects of Polypodium leucotomos on membrane integrity, lipid peroxidation, and expression of elastin and matrixmetalloproteinase-1 in ultraviolet radiation exposed fibroblasts, and keratinocytes," Journal of Dermatological Science, vol. 32, no. 1, pp. 1-9, 2003.

[14] S. Gonzalez, Y. Gilaberte, and N. Philips, "Mechanistic insights in the use of a Polypodium leucotomos extract as an oral and topical photoprotective agent," Photochemical and Photobiological Sciences, vol. 9, no. 4, pp. 559-563, 2010.

[15] S. Gonzalez, N. Philips, and Y. Gilaberte, "Photoprotection: update in UV filter molecules, the "new wave" of sunscreens," Dermatology and Venereology, vol. 145, no. 4, pp. 515-523, 2010.

[16] S. Astner, A. Wu, J. Chen et al., "Dietary lutein/zeaxanthin partially reduces photoaging and photocarcinogenesis in chronically UVB-irradiated Skh-1 hairless mice," Skin Pharmacology and Physiology, vol. 20, no. 6, pp. 283-291, 2007.

[17] N. Philips, T. Keller, C. Hendrix et al., "Regulation of the extracellular matrix remodeling by lutein in dermal fibroblasts, melanoma cells, and ultraviolet radiation exposed fibroblasts," Archives of Dermatological Research, vol. 299, no. 8, pp. 373379, 2007.

[18] N. Philips, M. Samuel, R. Arena et al., "Direct inhibition of elastase and matrixmetalloproteinases and stimulation of biosynthesis of fibrillar collagens, elastin, and fibrillins by xanthohumol," Journal of Cosmetic Science, vol. 61, no. 2, pp. 125-132, 2010.

[19] N. Philips, "Reciprocal effects of ascorbate on cancer cell growth and the expression of matrix metalloproteinases and transforming growth factor-beta: modulation by gene silencing or P. leucotomos," in Hanbook of Vitamin C Research: Daily Requirements, Dietary Sources, and Adverse Effects, $\mathrm{H}$. Kucharski and Julek, Eds., Nova Science, 2009.

[20] J. E. Rundhaug, "Matrix metalloproteinases, angiogenesis and cancer," Clinical Cancer Research, vol. 9, no. 2, pp. 551-554, 2003.

[21] A. Noël, M. Jost, and E. Maquoi, "Matrix metalloproteinases at cancer tumor-host interface," Seminars in Cell and Developmental Biology, vol. 19, no. 1, pp. 52-60, 2008.

[22] L. A. Liotta, K. Tryggvason, S. Garbisa, I. Hart, C. M. Foltz, and S. Shafie, "Metastatic potential correlates with enzymatic degradation of basement membrane collagen," Nature, vol. 284, no. 5751, pp. 67-68, 1980.

[23] N. Philips, T. Keller, and C. Holmes, "Reciprocal effects of ascorbate on cancer cell growth and the expression of matrix 
metalloproteinases and transforming growth factor- $\beta$," Cancer Letters, vol. 256, no. 1, pp. 49-55, 2007.

[24] N. Philips, L. Dulaj, and T.. Upadhya, "Growth inhibitory mechanism of ascorbate and counteraction of its matrix metalloproteinases-1 and transforming growth factor-beta stimulation by gene silencing or P. leucotomos," Anticancer Research, vol. 29, pp. 3233-3238, 2009.

[25] N. Philips, M. Tuason, T. Chang, Y. Lin, M. Tahir, and S. G. Rodriguez, "Differential effects of ceramide on cell viability and extracellular matrix remodeling in keratinocytes and fibroblasts," Skin Pharmacology and Physiology, vol. 22, no. 3, pp. 151-157, 2009.

[26] N. Buisson-Legendre, H. Emonard, P. Bernard, and W. Hornebeck, "Relationship between cell-associated matrix metalloproteinase 9 and psoriatic keratinocyte growth," Journal of Investigative Dermatology, vol. 115, no. 2, pp. 213-218, 2000.

[27] J. Arikawa, M. Ishibashi, M. Kawashima, Y. Takagi, Y. Ichikawa, and G. Imokawa, "Decreased levels of sphingosine, a natural antimicrobial agent, may be associated with vulnerability of the stratum corneum from patients with atopic dermatitis to colonization by Staphylococcus aureus," Journal of Investigative Dermatology, vol. 119, no. 2, pp. 433-439, 2002.

[28] E. Tschachler, "Psoriasis: the epidermal component," Clinics in Dermatology, vol. 25, no. 6, pp. 589-595, 2007.

[29] Y. Ohnishi, N. Okino, M. Ito, and S. Imayama, "Ceramidase activity in bacterial skin flora as a possible cause of ceramide deficiency in atopic dermatitis," Clinical and Diagnostic Laboratory Immunology, vol. 6, no. 1, pp. 101-104, 1999.

[30] B. L. Lew, Y. Cho, J. Kim, W. Y. Sim, and N. I. Kim, “Ceramides and cell signaling molecules in psoriatic epidermis: reduced levels of ceramides, PKC- $\alpha$, and JNK," Journal of Korean Medical Science, vol. 21, no. 1, pp. 95-99, 2006.

[31] N. Philips, H. Hwang, S. Chauhan, D. Leonardi, and S. Gonzalez, "Stimulation of cell proliferation and expression of matrixmetalloproteinase- 1 and interluekin- 8 genes in dermal fibroblasts by copper," Connective Tissue Research, vol. 51, no. 3, pp. 224-229, 2010.

[32] N. Philips, R. Arena, and S. Yarlagadda, "Inhibition of ultraviolet radiation mediated extracellular matrix remodeling in fibroblasts by transforming growth factor," Bios, vol. 80, pp. 20-24, 2009.

[33] N. Philips, T. Keller, and S. Gonzalez, “TGF $\beta$-like regulation of matrix metalloproteinases by anti-transforming growth factor- $\beta$, and anti-transforming growth factor- $\beta 1$ antibodies in dermal fibroblasts: implications for wound healing," Wound Repair and Regeneration, vol. 12, no. 1, pp. 53-59, 2004.

[34] A. Brieva, N. Philips, R. Tejedor et al., "Molecular basis for the regenerative properties of a secretion of the mollusk Cryptomphalus aspersa," Skin Pharmacology and Physiology, vol. 21, no. 1, pp. 15-22, 2008.

[35] Z. Ahmed, A. Briden, S. Hall, and R. A. Brown, "Stabilisation of cables of fibronectin with micromolar concentrations of copper: in vitro cell substrate properties," Biomaterials, vol. 25, no. 5, pp. 803-812, 2004. 

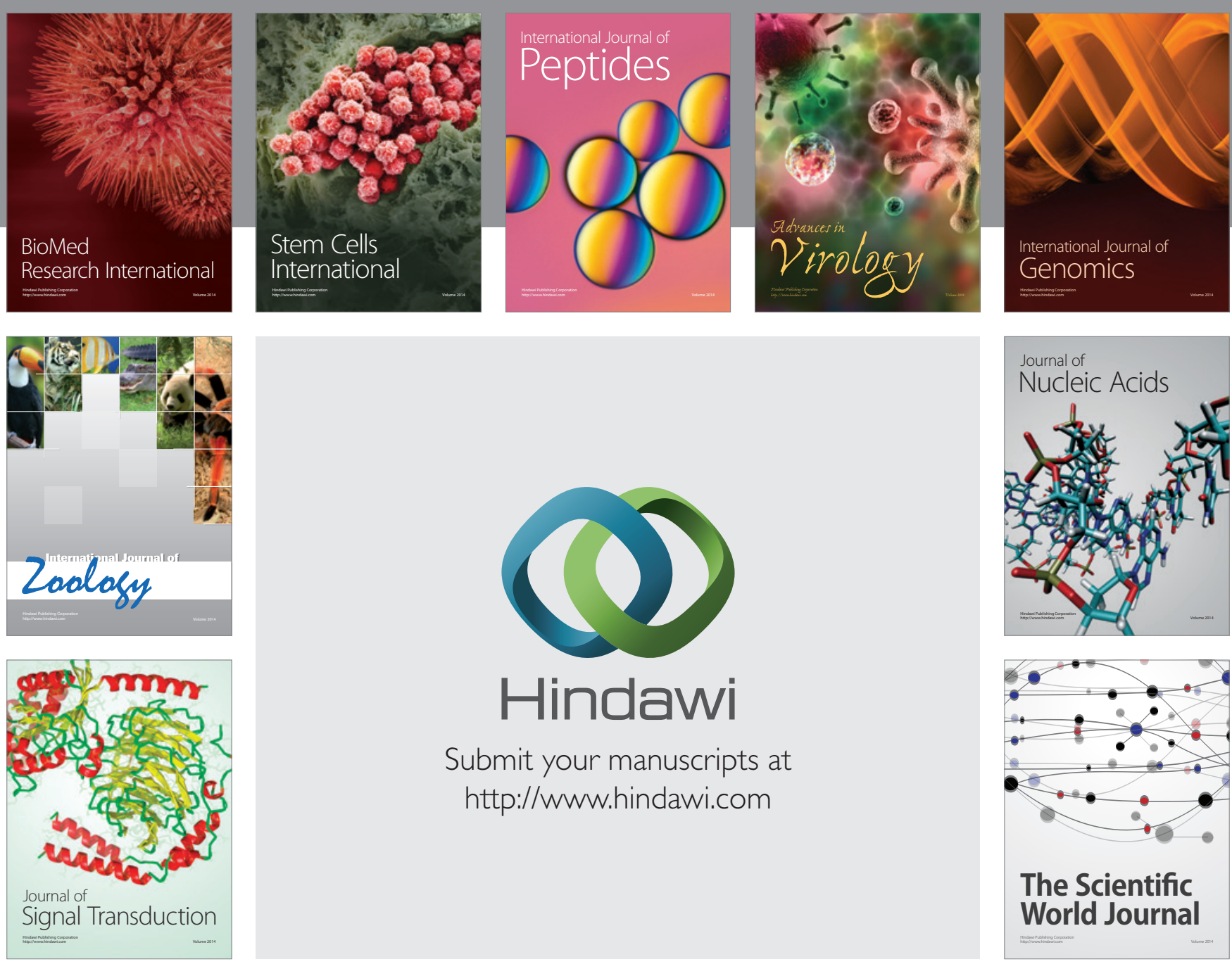

Submit your manuscripts at

http://www.hindawi.com
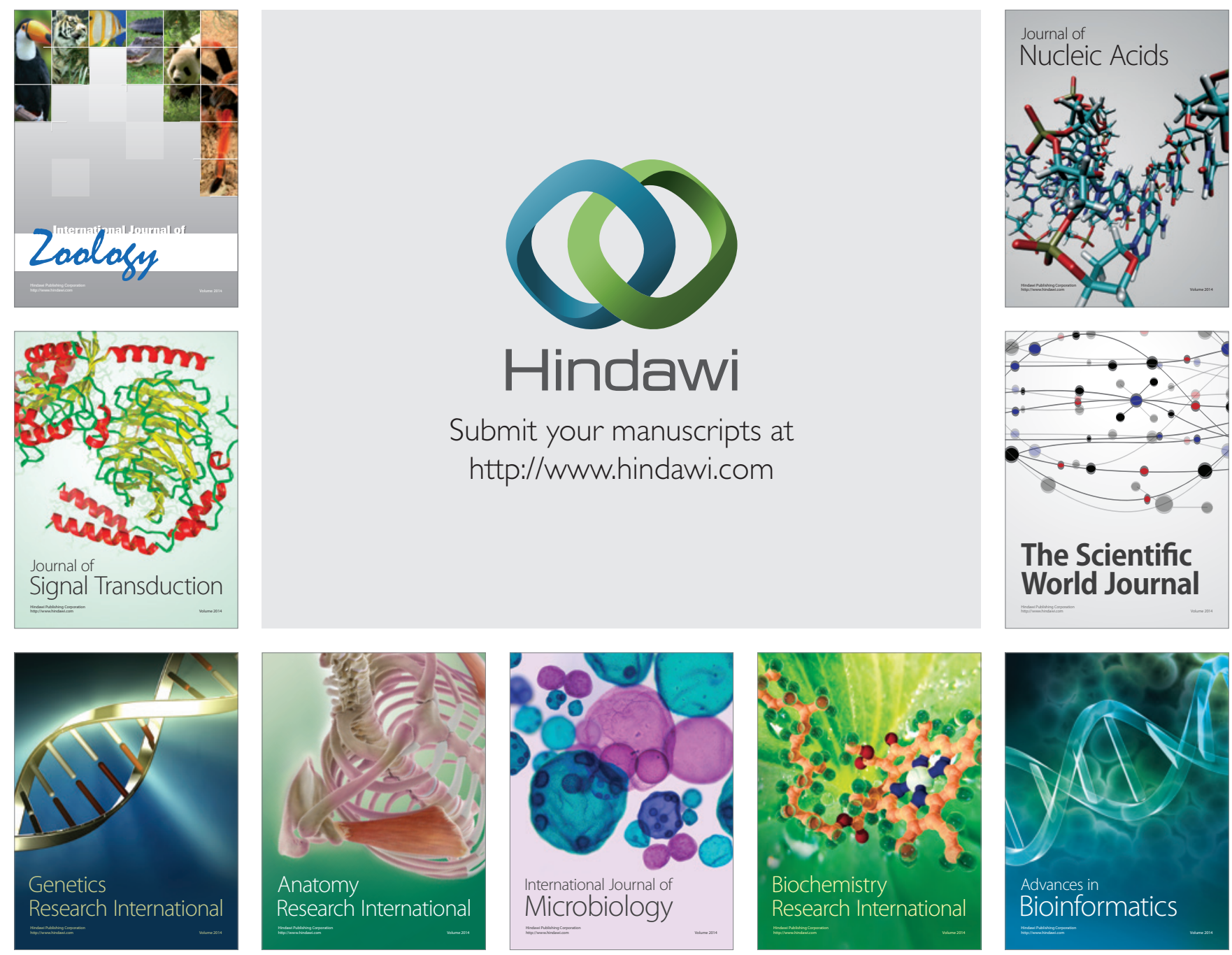

The Scientific World Journal
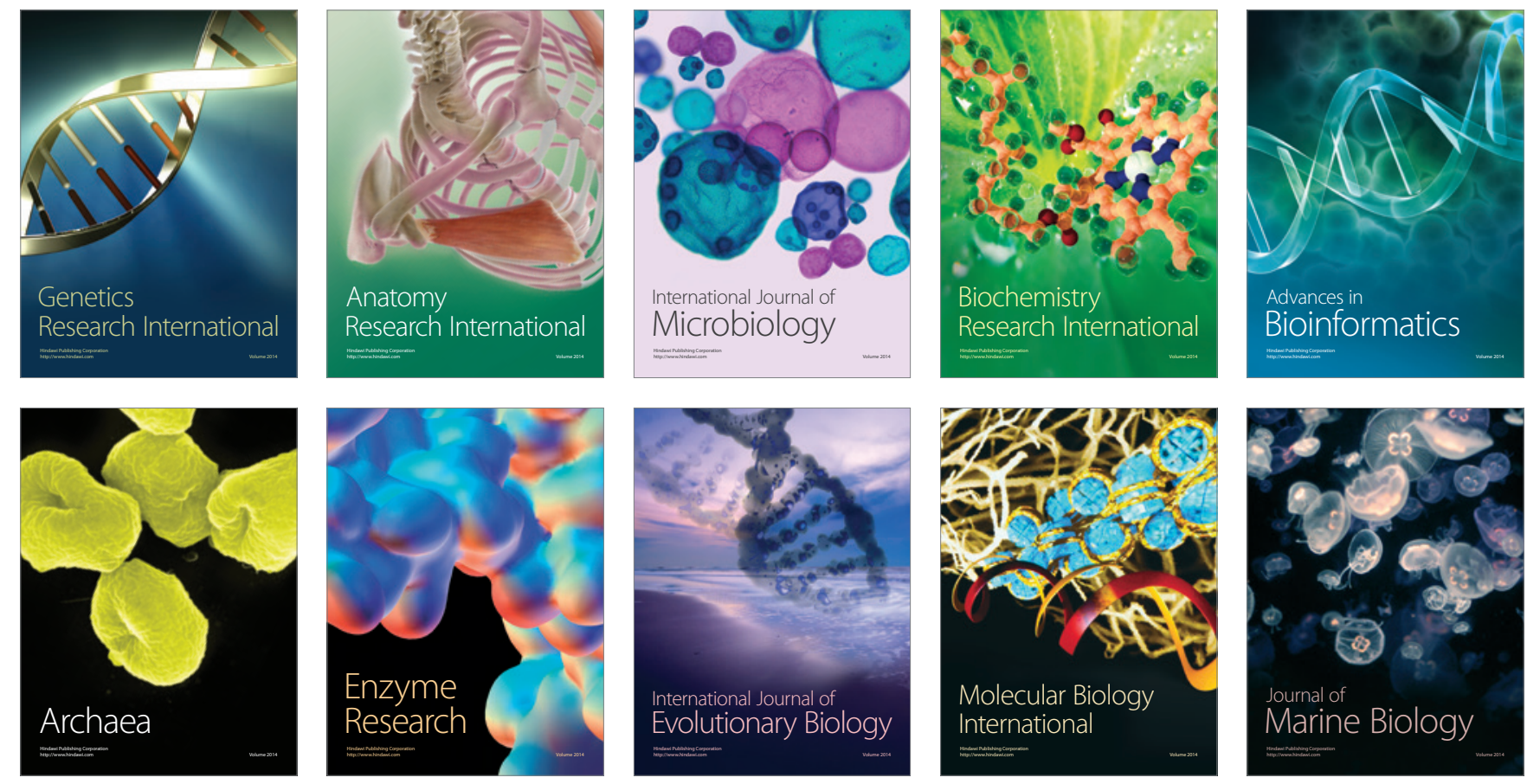